\title{
Research on the Information Management of University Stadiums Based on Digital Twin Technology
}

\author{
Peng Hou ${ }^{1}$, Shaoyong Yu ${ }^{1}$, Yaowei Song ${ }^{2}$ \\ ${ }^{1}$ Department of Physical Education, Xidian University, Xi'an 710071, Shaanxi, China \\ ${ }^{2}$ Institute of Physical Education, Xi'an Physical Education University, Xi'an 710068, Shaanxi, China \\ Email: houpeng@xidian.edu.cn
}

\begin{abstract}
In order to promote the information, intelligent and standardized management of the stadiums in colleges and universities, and to provide better services for the stadiums of the 14th National Games of China in Shaanxi Province, this article explores the information management of stadiums in colleges and universities based on digital twin technology. Using digital twin technology to copy university stadiums in virtual space, as the mirroring, mapping, simulation and assistance of real stadiums, this article provides digital basic support for smart stadium planning, construction, and operation management, including comprehensive data simulation and operation management. This article puts forward the design conception of intelligent stadiums as well as the information function, social function, game-watching service function, data analysis function and so on.
\end{abstract}

Keywords: digital twin technology, stadiums, information management

\section{Introduction}

The application of science and technology in the sports field is gradually moving from behind the scenes to the front of the stage. Under the background of the strategy of "network power", China has issued "Internet + " and other national development and implementation strategies. How to realize the interaction and integration between the manufacturing physical world and the information world has become the core bottleneck in the development of informationization and intelligence of stadiums in China ${ }^{[1]}$.

Colleges and universities are the important carriers of stadiums in China. According to the calculation of the General Administration of Sport of China in 2008, among the 1.04 million stadiums of various kinds across the country, the stadiums in the education system account for about $65.6 \%{ }^{[2]}$. The article focuses on exploring the establishment of an information management platform for university stadiums based on digital twin technology, and realizing the information and intelligent management of college sports venues, so as to promote the more efficient management of university stadiums and the opening of large-scale events, and optimize the resource allocation of stadiums .

\section{The teaching and management status of university stadiums}

\subsection{The teaching status of university stadiums}

From a specific point of view, at the beginning of the semester, the teaching staff and stadium management personnel responsible for arranging classes should distribute each teaching site in the school to each class and time period, and report to the Academic Affairs Office and departments in time. They communicate extensively with colleges and counselors through interviews, surveys or seminars to grasp the needs of physical education teaching, so as to accurately grasp the use of stadiums and deal with some unexpected problems. This lays the foundation for the standardization, high-efficiency and scientificization of stadium management ${ }^{[3]}$.

\subsection{Management status of university stadiums}

Usually when large-scale competitions or activities are held, there is a large flow of people, and safety is the top priority. It is necessary not only to formulate a safety plan and conduct drills in advance, but also to comprehensively inspect the fire-fighting facilities ${ }^{[3]}$. To improve the effectiveness of management, regional management is often adopted. To host an event, it is necessary to set up VIP area, media area, audience area, competition area, referee area, athlete rest area, etc., and implement different pass area certificates for different people to strengthen classification management. But at the same time, there are problems such as shortage of workforce and increased depletion of public facilities, which can easily cause serious economic losses. 


\section{Related theoretical and applied research}

The intelligent construction of stadiums in China has been developed for less than 20 years. The first one to realize the intelligentization of stadiums was the 80,000 people stadium built in Shanghai in $1997^{[4]}$. The 2008 Olympic stadiums are the outstanding representatives of intelligent buildings in the world today, and their intelligence is also called digital ${ }^{[5]}$. The information management mode of university stadiums is a novel management mode, which is still in its infancy, and it is rare in the management of university stadiums ${ }^{[6]}$.

Digital twin technology creates a virtual model of a physical entity in a digital way, uses data to simulate the behavior of a physical entity in the real environment, and adds or expands new capabilities for the physical entity through virtualreal interactive feedback, data fusion analysis, and decision-making iteration optimization. It is a technology that integrates multi-physics, multi-scale, multi-disciplinary attributes, has real-time synchronization, faithful mapping, and high fidelity characteristics, and can realize the interaction and integration of the physical world and the information world ${ }^{[1,7-8]}$.

The concept of digital twin can be traced back to 1969. Its clear concept is generally believed to have been proposed by Professor Michael Grieves of the United States in 2003. It was called "virtual digital expression equivalent to physical products" at that time. The concept was not taken seriously ${ }^{[9-10]}$. After this, Lockheed Martin listed the digital twin as the top 6 top technologies in the future defense and aerospace industry in November 2017. In the same year, the China Association for Science and Technology Intelligent Manufacturing Academic Consortium listed the digital twin as one of the world's top ten technological advances in intelligent manufacturing at the World Intelligent Manufacturing Conference. The academic community has mainly carried out related research on the modeling of digital twin ${ }^{[1]}$, cyber-physical integration ${ }^{[12]}$, interaction and collaboration ${ }^{[13]}$, and service applications ${ }^{[14]}$.

\section{Related analysis of information management of university stadiums}

\subsection{Operating status of university stadiums}

Take the Invengo Gymnasium of Xidian University as an example, as is shown in Figure 1. Most of these stadiums have both teaching and industrial management functions. Whether it comes to a teaching or a large-scale event, when there are tens of thousands of people every day, a safe and smooth management system is still the top priority. The planning of stadium facilities, stadium management, safety management and other issues caused the operation managers to be exhausted. 


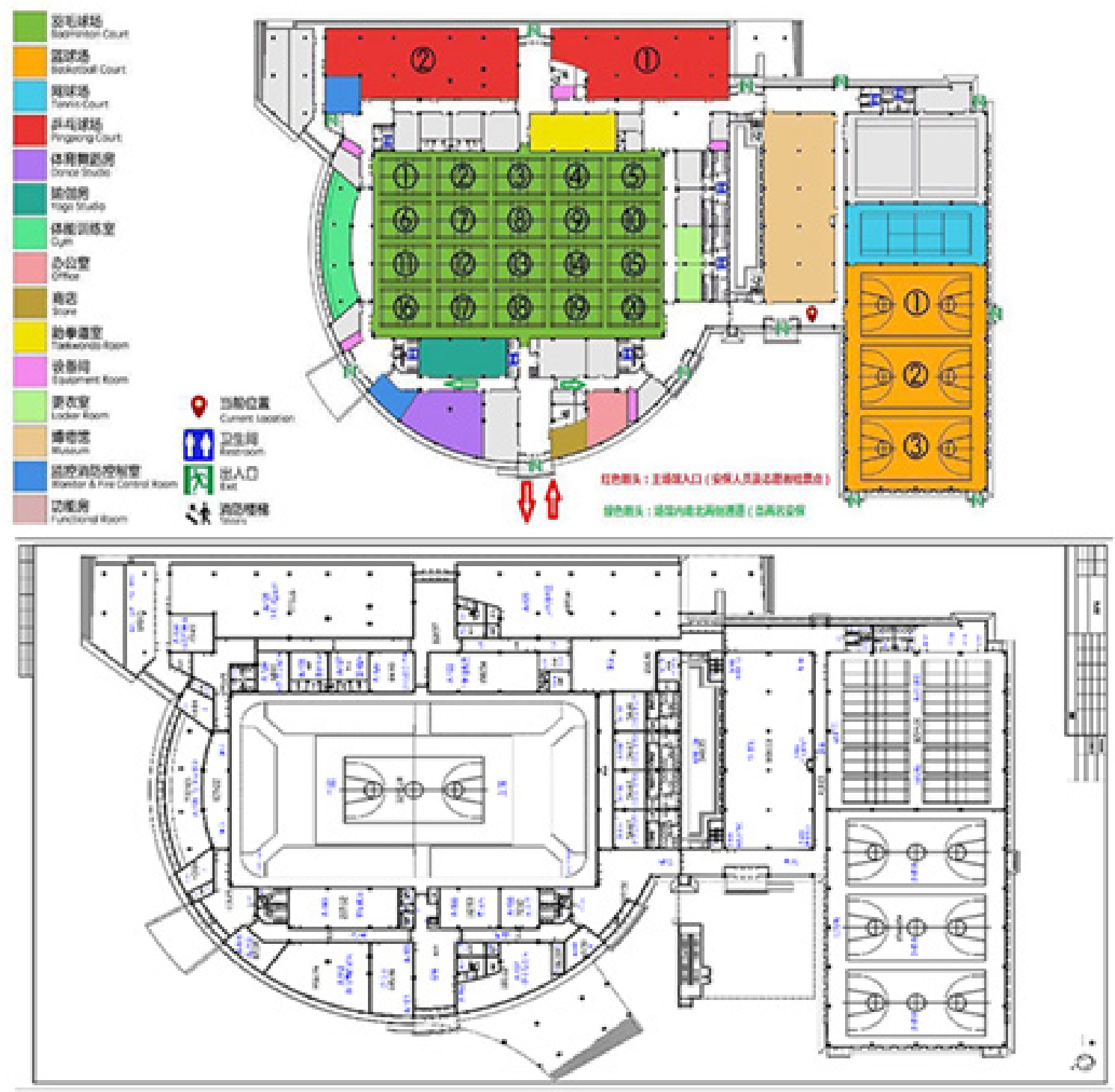

Figure 1. Floor plan of Invengo Gymnasium of Xidian University

Note: Red haircut - entrance to the main stadium (security personnel and volunteer check-in points); green haircut — the north and south sides of the stadium (two security guards each).

Through the construction of a digital twin, the real-time and multi-dimensional digital presentation of various target building areas and facilities in the stadium is carried out. The various scenes in the Invengo Gymnasium are accurately restored, and the various elements in the scene are classified and associated. It is integrated with the corresponding physical objects, creating a virtual-reality integrated dynamic service decision-making management platform.

\subsection{Network conditions of digital twin}

One of the basic conditions for the realization of digital twin technology is the generation and recording of information. For stadiums, it is first necessary to have smart objects that enable digital twins to be realized, software suitable for the needs of users and daily management of stadium managers, and digital infrastructure that contains multiple layers and provides a monitoring environment for the software. Multi-layer network structure is indispensable; the information transmission between equipment needs to be accurate and efficient at the same time.

In addition, a variety of sensors need to be deployed to comprehensively consider multiple aspects of functions to ensure the accuracy, real-time, and trace ability of data.

The application of wireless network provides a more solid network guarantee for the digital twin. The end-to-end 
transmission capability of $5 \mathrm{G}$ technology will enhance the integration capability of various sensor data.

\subsection{Requirements for teaching system}

The construction of an intelligent physical education system is an urgent need for university stadiums based on daily teaching use and maintenance. College public physical education is an indispensable course for students at the university level, but there are long-standing problems such as backward teaching mode, outdated teaching materials, and insufficient innovation ability of teachers, which cannot arouse students' interest. This requires full consideration of interest factors when constructing an intelligent teaching system, changing the inherent thinking of "teaching is learning" in the past, and making full use of digitalization and information technology to collect, transmit, and analyze the physical and physical data of students. The selection of training items and the formulation of exercise programs provide accurate data support and guarantee.

\subsection{Requirements for safety management system}

The safety management of university stadiums is a very special and important part of the safety management of colleges and universities. In the new era, the scale of stadium construction continues to expand; equipment replacement is frequent; the number of students increases, which puts huge pressure on stadium safety management. Therefore, based on the stadium safety management system, the following aspects need to be considered. (1) The contradiction between the increasing area of stadiums, the increase of students and the shortage of management personnel; (2) the large number of sports facilities and equipment, the variety of them, the difficulty of maintenance, and the objectively existing possibility of safety accidents caused by equipment failure or damage; (3) human-made uncertain factors continue to increase, and personnel management are difficult; (4) the ability to respond to emergencies is insufficient, and the ability of disaster early warning and timely disposal is weak.

\section{Functional design of stadium digital twin system}

\subsection{Simulation design of comprehensive data of university stadiums}

Based on hierarchical management and multi-source data and analysis system management, the integrated comprehensive management and control of stadiums are realized. It covers basic spatial data (three-dimensional realworld images of stadiums, terrain, landmarks, etc.) and dynamic monitoring data (dynamic distribution of students, venue access data, sports equipment usage, etc.) to create a standardized, digitized, and visualized integrated data and service management system. Under the premise of ensuring the validity of real-time data, through real-time strategy demonstration and dynamic simulation visual forecasting, the screening and optimization of routine management and emergency handling plans are realized.

\subsection{Simulation design for the operation and management of university stadiums}

In the process of operation and management, the managers of university stadiums use various sensors and intelligent operation and maintenance terminals installed in the stadiums to collect real-time data to build a stadium-based CIM digital model. Based on this, advanced maintenance of equipment, virtual simulation and deduction of emergency response, disaster prevention and early warning, and flow of people in large-scale events have been realized, making stadium operations more intelligent and technological, and ensuring the safety and efficiency of daily operations.

\subsection{Functional design of digital twin technology in stadiums}

This article discusses the information management of university stadiums from the information function, social function, game-watching service function, teaching data analysis function, security rehearsal function and risk warning function ${ }^{[15]}$, as shown in Figure 2. 


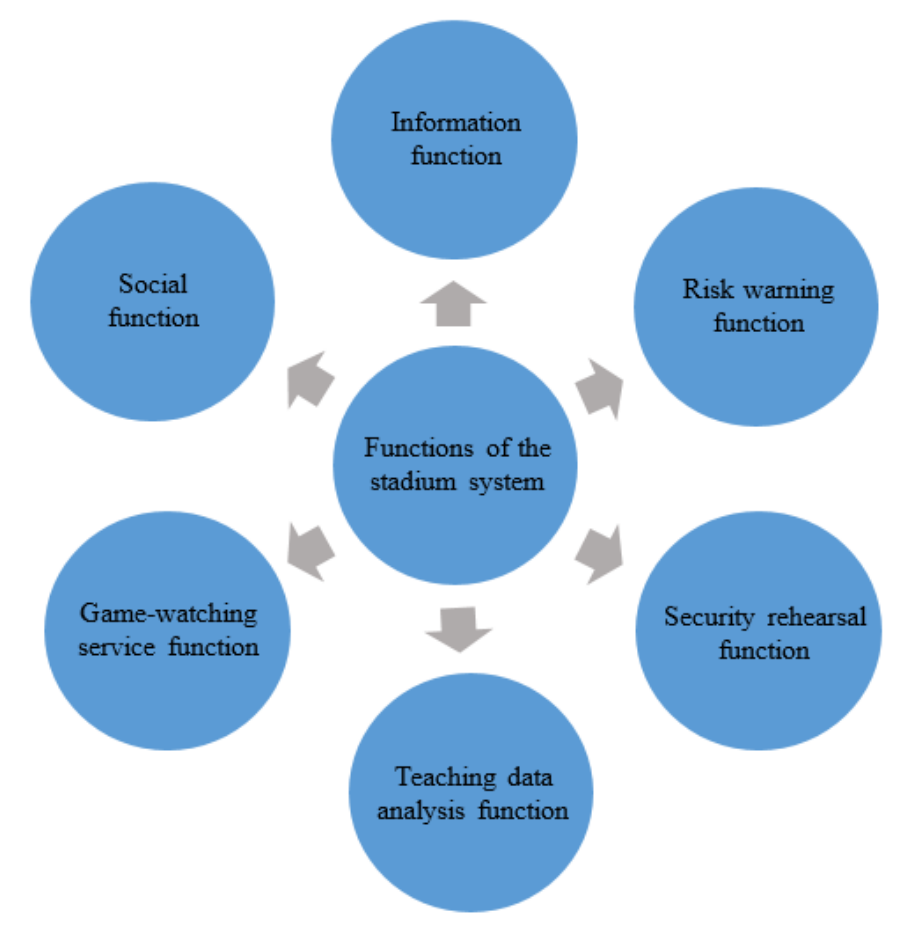

Figure 2. Construction of information management scheme for university stadiums

\subsubsection{Information function}

China Internet Network Information Center (CNNIC) released the 45th "Statistical Report on China's Internet Development Status" in August 2019. As shown in Table 1. A huge group of netizens constitutes China's booming online consumer market and has also laid a solid user base for the development of the digital economy. Correspondingly, as network solution providers such as Huawei and ZTE have made great efforts in the wireless network coverage of stadiums, the informatization capabilities of stadiums have been improved to a large extent, and the digital operation and management capabilities of stadiums are also being strengthened.

Table 1. The status of China Internet development in 2019 and early 2020

\begin{tabular}{cc}
\hline Items & Parameters \\
\hline The number of netizens & 904 million \\
The number of mobile Internet users & 897 million \\
The number of IPv6 adresses & 50877 blocks/32 \\
The number of IPv4 adresses & 387.51 million \\
The total number of domain names & 50.94 million \\
The number of ".CN" domain names & 22.43 million \\
The number of websites & 4.97 million
\end{tabular}

\subsubsection{Social function}

University stadiums take providing service for students' daily sports activities as the main service function, and have natural social attributes. The university stadium management system also needs to have certain social functions to increase the two-way interaction between students, so as to achieve the purpose of improving experience and efficiency. Corresponding function points include virtual community, event interaction, live broadcast, chat and friendship, service consultation, etc.

\subsubsection{Game-watching service function}

University stadiums need to meet people's needs of all aspects in the process of watching games. University stadiums should design a human-based event service system, incorporating factors such as weather, personnel flow, and noise into service decisions. Through the use of H5, app and WeChat mini programs, interactive services with a high degree of participation such as WeChat message wall and quiz games are added to the game watching. In addition, the introduction 
of artificial intelligence and wearable devices also uses wireless networks and 5G technology to enrich the content of the event, allowing the audience to obtain a multi-angle and all-round service experience.

\subsubsection{Teaching data analysis function}

Teaching data analysis is one of the important functions of the digital twin system of university stadiums. Through the visual display of data on the number of participants in the classroom, exercise intensity, and physical fitness improvement of students by installation of smart sensors, teachers can easily analyze students' interest in physical education subjects, adjust the curriculum structure in time, and improve student participation in physical education classes. Through data comparison of different teachers' teaching, teaching items and types, universities can develop attractive physical education courses and optimize the design of physical education classroom subjects to achieve the goal of enhancing students' physical fitness and promoting the coordinated development of students' body and mind.

\subsubsection{Risk warning function}

Risk warning management is to dynamically assess the risk of safety accidents in stadiums based on the collected data. When the accident has not occurred and there is a greater risk of occurrence or when the early accident occurs, the safety assessment and strategy analysis are carried out to seek the best safety solution. When a sudden emergency accident occurs in a stadium, the best escape route is given by analyzing the cause and consequences of the accident. At the same time, it also provides rescue workers with rescue information such as the health status, number and location of the people trapped at the scene, so as to improve the risk control ability and the ability to deal with emergency accidents.

\subsection{Discussion}

Digital twin technology brings innovative solutions to the comprehensive management of university stadiums. Digital twin university stadiums, which complete the mapping in the virtual space and guide the physical world, have considerable advantages in daily teaching and event management and operation.

Digital twin university stadiums are an important part of the informatization construction of new smart university stadiums. In the exploration of the construction of smart university stadiums, the adoption of digital twin technology provides a new technology integration point and development direction for university smart management. The characteristics of virtual and physical integration and precise mapping of digital twin university stadiums will also impose more stringent requirements on information transmission capabilities.

\section{Conclusion}

This paper combs the exploration of the innovative application of information technology in stadiums in recent years, and draws on the current successful experience of smart stadiums in infrastructure construction, sensor layout, and information transmission. The various functions designed according to "service requirements for watching games" provide technical support for the socialized operation of university stadiums and clarify ideas for the informatization construction of various stadiums. Relevant government departments also need to strengthen their support for informatized smart stadiums to ensure the proportion of stadiums' investment in smart operations, so as to form a new situation of tripartite cooperation and win-win technology application among the government, schools and enterprises.

\section{Acknowledgments}

The article is supported by: Sports Philosophy and Social Science Research Project of the State Sports General Administration (project number: 2370SS16107); Shaanxi Provincial Social Science Foundation Project (project number: 2016Q010); Xidian University Humanities and Information Science Cross Special Funding Project (project number: RW180177).

\section{References}

[1] Tao Fei, Liu Weiran, Liu Jianhua, et al. Digital twin and its application exploration. Computer Integrated Manufacturing System. 2018; 24(01): 1-18.

[2] State Sports General Administration. 50 Years of Sports in New China. Beijing: People's Sports Publishing House; 2009.

[3] Huo Jianxin, Li Fu. Research on Comprehensive Utilization of Gymnasiums in Colleges and Universities in China. Journal of Tianjin Institute of Physical Education. 2007; (02): 179-181.

[4] Liu Huiming. Talking about the intelligent construction of stadiums in China — an interview with senior engineer 
Shu Shiliang of the Sports Information Center of the State Sports General Administration. Intelligent Building. 2004; (7): 64-66, 69.

[5] Bi Jinbo. Discussion on the management and operation of digital stadiums. Intelligent Building and Urban Information. 2008; (5): 16-19.

[6] Zeng Xiaosong, Chen Xiaorong, Li Wang, Chen Binhong. Effective management of the stadiums of Shenzhen University. Journal of Physical Education. 2016;23(01): 76-79.

[7] Tao Fei, Zhang Meng. Digital twin shop-floor: a new shop-floor paradigm towards smart manufacturing. IEEE Access. 2017; 5: 20418-20427.

[8] Li Xin, Liu Xiu, Wan Xinxin. Overview of Digital Twin Application and Security Development. Journal of System Simulation. 2019; 31(03): 385-392.

[9] Grieves M. Product lifecycle management: the new paradigm for enterprises. International Journal of Product Development. 2005; 2(1/2): 71.

[10] Grieves M. Product lifecycle management — driving the next generation of lean thinking. New York: McGraw-Hill Companies; 2006.

[11] Aitor M, Gorka V, Aitor A, et al. Virtualisation process of a sheet metal punching machine within the Industry 4.0 vision. Internation Journal on Interactive Design and Manufacturing; 2017, 11(2): 365-373.

[12] Tao Fei, Cheng Ying, Cheng Jiangfeng, et al. Theory and technology of cyber-physical fusion in digital twin workshop. Computer Integrated Manufacturing System. 2017; 23(08): 1603-1611.

[13] Uhlemann T H J, Schock C, Lehmann C, et al. The digital twin: demonstrating the potential of real timedata acquisition in production systems. Procedia Manufacturing. 2017; 9: 113-120.

[14] Bazilevsy, Deng X, Korobenko A, et al. Isogeometric fatigue damage prediction in large-scale composite structures driven by dynamic sensor data. Journal of Applied Mechanics. 2015; 82(9): 091008-1-12.

[15] Zhang Shaoqiong. Construction of information management platform for college stadiums and gymnasiums under the background of Internet +. Electronic Technology and Software Engineering. 2020; (02): 232-233. 